\title{
Single versus multi-dose intra-articular injection of platelet rich plasma in early stages of osteoarthritis of the knee: A single-blind, randomized, superiority trial
}

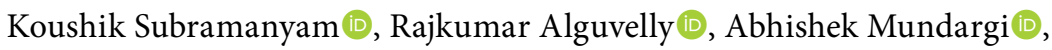 \\ Prakash Khanchandani 1 \\ Department of Orthopaedics, Sri Sathya Sai Institute of Higher Medical Sciences - Prashanthigram, Puttaparthi, Andhra Pradesh, India
}

\begin{abstract}
Objectives: This study aims to compare the effectiveness of single, double, and triple doses of intra-articular (IA) platelet rich plasma (PRP) in early stages of osteoarthritis (OA) of the knee.

Patients and methods: This single-blind, randomized, superiority trial included a total of 180 knees of 90 patients (22 males, 68 females; mean age: 47.9 years; range, 36 to 60 years) with bilateral OA knee of Kellgren-Lawrence Grade 1-2 between May 2017 and December 2018. The patients were randomized (30 in each group) to receive single, double, or triple doses of IA PRP (two weeks apart in repeat injections). The outcome measures were Visual Analog Scale, International Knee Documentation Committee Score, Knee Injury and Osteoarthritis Outcome Score, and Tegner Lysholm Knee Score. The assessor of outcome was blinded. The scores were collected before intervention and at six weeks, three months, six months, and one year after the intervention.

Results: All patients completed the study. All three groups were comparable with respect to demographic and disease characteristics. All four scores were comparable among the three groups before intervention and at six weeks, three months, and six months. However, at one-year follow-up, the three-dose group showed superiority to others in terms of all four scores. All three groups showed improvement until six months and deterioration thereafter, which was only marginal in the three-dose group. All groups showed a statistically significant improvement of scores compared to baseline scores at one year. There were no major complications.
\end{abstract}

Conclusion: The IA administration of three doses of PRP yields superior outcome to single and double doses at the end of one year. Repeat doses are probably needed to sustain the benefit achieved at one year.

Keywords: Knee osteoarthritis, orthobiologics, platelet rich plasma.

Knee osteoarthritis (OA) affects more than $20 \%$ of individuals older than 45 years and is the second most common cause of work performance loss after low back pain. ${ }^{1}$ With the increasing life expectancy, the requirement for total knee arthroplasty surgeries is on the rise, adding significant economic burden to patients and healthcare systems. ${ }^{2}$ In search of more conservative and biological approaches for treatment of this chronic progressive disease, intra-articular (IA) delivery of platelet rich plasma (PRP) appears to be a promising modality. The PRP is thought to deliver a large pool of growth factors and proteins implicated in tissue repairing mechanisms. ${ }^{3}$ This intervention is thought to, at least, save time and postpone total knee arthroplasty in young patients, avoiding revision surgeries in the future. ${ }^{4}$

Received: August 28, 2020 Accepted: November 05, 2020 Published online: January 14, 2021

Correspondence: Koushik Subramanyam, MD. Department of Orthopaedics, Sri Sathya Sai Institute of Higher Medical Sciences - Prashanthigram, 515134 Puttaparthi, Andhra Pradesh, India. Tel: +919491629620 e-mail: drkoushik@hotmail.com

Subramanyam K, Alguvelly R, Mundargi A, Khanchandani P. Single versus multi-dose intra-articular injection of platelet rich plasma in early stages of osteoarthritis of the knee: A single-blind, randomized, superiority trial. Arch Rheumatol 2021;36(3):326-334. 
Although IA PRP administration has resulted in improvement of pain and function in OA knee, the optimal dose still remains uncertain. The studies available have used one to three doses of IA PRP. To date, no standard recommendation has been made for IA PRP administration. ${ }^{5} \mathrm{~A}$ recent meta-analysis on comparison of single versus multiple IA injections of PRP in OA knee concluded that multiple injections seemed to be superior for joint functionality after six months, although there might not be any difference in the short term. ${ }^{6}$ However, the study categorically concluded that the available evidence was still insufficient and future research on this specific topic were needed.

In spite of the suggestion from literature that multiple doses would be superior to single dose, it is not clear how much is "multiple". Until date, there are only six randomized-controlled trials (RCTs) comparing dosage regimens of IA PRP in OA knee. ${ }^{7-12}$ Five of them compared the results between two dosage schedules-either single versus double $e^{7,12}$ or single versus triple..$^{8-10}$ Only one among them compared single versus double versus triple doses of IA PRP. ${ }^{11}$ In addition, there is a retrospective study comparing single versus double versus triple doses of IA PRP. ${ }^{13}$ Among the two studies which compared three dosages of PRP, one concluded that two doses would be optimum. ${ }^{11}$ However, the other study reported that two doses would be insufficient and recommended three doses. ${ }^{13}$ The only RCT on three doses enrolled patients with advanced stage of OA knee, and there is no RCT comparing three doses of IA PRP in early stages of OA knee. In the present study, therefore, we aimed to compare the effectiveness of single, double, and triple doses of IA PRP in early stages of OA of the knee.

\section{PATIENTS AND METHODS}

This single-blind, three-arm, prospective, randomized, superiority trial (1:1:1 parallel allocation) was conducted at the Department of Orthopedics and Traumatology of a tertiary level charitable super-speciality hospital between May 2017 and December 2018. Change in the Visual Analog Scale (VAS), being the primary outcome was taken as the basis for calculating sample size, based on the previous study by Patel et al. ${ }^{7}$ The assumed study power was set to $80 \%$ (beta $=0.2$ ), false-positive rate was 5\% (alpha $=0.05$ ), the predicted difference of mean VAS between groups was 1.5 , and the standard deviation was 0.6. Using these parameters, and adjusting our alpha for multiple comparisons, we required 25 patients per treatment arm. To account for loss to follow-up, we decided to recruit 30 patients per each arm, making it a total sample size of 90 . A written informed consent was obtained from each participant. The study protocol was approved by Sri Sathya Sai Institute of Higher Medical Sciences - Prasanthigram Ethics Committee (SSSIHMS$\mathrm{PG} / \mathrm{ACAD} / 15$ ) and was registered in the Clinical Trial Registry India (CTRI/2017/04/008406) prior to commencement of the study. The study was conducted in accordance with the principles of the Declaration of Helsinki.

All patients aged 35 to 60 years presenting outpatient department with bilateral OA of the knee were screened for the study. Bilateral $\mathrm{OA}$ of the knee was diagnosed according to the American College of Rheumatology (ACR) criteria $^{14}$ and radiological staging was done according to the Kellgren-Lawrence (KL) system..$^{15}$ A total of 180 knees of 90 patients (22 males, 68 females; mean age: 47.9 years; range 36 to 60 years) with bilateral OA knee of Kellgren-Lawrence Grade 1-2 were included in the study. Exclusion criteria were KL Grade 3-4, knee instability, malalignment more than $5^{\circ}$, patellar maltracking, OA secondary to inflammatory/metabolic diseases, coexisting back pain, history of infection/steroid injection/ arthroscopy/surgery to knee, anticoagulant use, malignancies, and poor general condition. It was ensured that the patients did not have non-steriodal anti-inflammatory drugs (NSAIDs) within one week and systemic steroids or immunomodulators within three months of PRP injection. Recruitment was stopped, when all treatment arms had 30 cases each. A Consolidated Standards of Reporting Trials (CONSORT) flow diagram showing the enrolment, allocation of treatment, and passage through the study is shown in Figure 1.

The patients were randomized into three groups by an independent allocator using block randomization with randomly mixed block sizes of two, four, and six, with concealed sizes of block. The allocator made random cards in sealed 


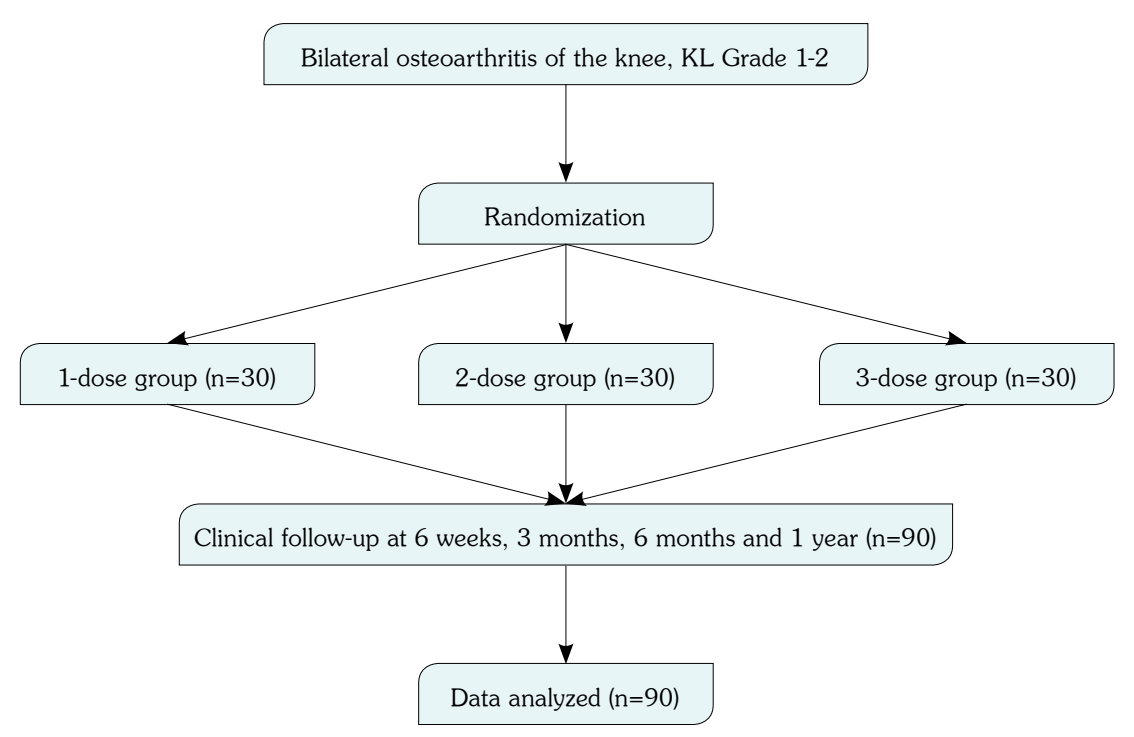

Figure 1. Study flow chart.

KL: Kellgren-Lawrence.

envelopes using computer-generated random numbers. A duplicate set was made in case individual code-breaking was required. During trial counselling and consenting, the sequentially numbered sealed envelope with the patient allocation was made available. Accordingly, the patients were divided into single, double, and triple dose groups. In the latter two groups, the repeat injections were given two weeks after the previous injection. All injections were performed by a single surgeon. Neither the patient nor the surgeon was blinded to the randomization. However, the outcome assessment was done by a dedicated observer who was not made aware as to which group the patient belonged to.

\section{PRP preparation}

All injections were given in the operating room under all sterile precautions and the PRP was prepared in the same sterile environment just prior to injection. The PRP was prepared by the protocol described by Gobbi et al. ${ }^{16}$ The technique is to centrifuge $8 \mathrm{~mL}$ of blood (with $2.7 \mathrm{~mL}$ ACD-A anticoagulant) from cubital vein for $5 \mathrm{~min}$ at $1,500 \mathrm{~g}$ centrifugal force and 3,500 revolutions per min. This yields $4 \mathrm{~mL}$ PRP with platelet recovery $80 \%$ (roughly twofold increase) and total leucocyte concentration below the normal level-specific granulocyte depletion $>95 \%$ (mostly mononuclear cells being recovered 75\% lymphocytes and 50\% monocytes). This system did not use a second centrifugation process. Prior to commencement of our study, we validated this protocol by preparing PRP from 15 healthy volunteers (after ensuring normal platelet and leucocyte counts) after informed consent. All samples fitted to the standards described by Gobbi et al. ${ }^{16}$ Leucocyte poor-PRP was obtained according to Dohan Ehrenfest classification, ${ }^{17}$ while it was $\mathrm{P} 2 \mathrm{Bb}$ as per the Platelets, Activation, White cells (PAW) classification. ${ }^{18}$

\section{Interventional procedure and follow-up}

For the injection, the patient was placed in a supine position with the knee in full extension. Under aseptic precautions, $4 \mathrm{~mL}$ of platelet concentrate was injected into a suprapatellar pouch through a superolateral approach with a 20-gauge needle after topical anesthesia. The knees were immobilized for 10 min after injection. The patients were discharged after $30 \mathrm{~min}$ of observation. During the follow-up period, NSAIDs were not allowed. Patients were instructed to use oral paracetamol $650 \mathrm{mg}$ as and when required for control of pain, if there was real need. Pain medication was stopped $48 \mathrm{~h}$ prior to all follow-up assessments. Prior to injection, all patients received a week-long session of training in home-based exercises to be continued regularly 
for an indefinite period. ${ }^{19}$ Life style modifications such as restriction of stair climbing, avoidance of squatting and cross-legged sitting, weight reduction (if relevant) and moderate exercises were advised and followed for compliance.

\section{Outcome measures}

Pain and functional assessment at five time points - pre-intervention, at six weeks, three months, six months, and one year of follow-up was done. The scores captured were as follows: VAS, ${ }^{20}$ International Knee Documentation Committee (IKDC) Score, ${ }^{21}$ Knee Injury and Osteoarthritis Outcome Score (KOOS), ${ }^{22}$ and Tegner Lysholm (TL) Knee Score. ${ }^{23}$ The VAS was scored from 0-10 (0-no pain, 10-worst pain) whereas the other three scores were from 0-100 (0-worst outcome, 100-best outcome). All patients had pre-intervention radiographs for categorization to KL Grades 1-2. All these were done by a single, dedicated, blinded observer. If the values were dissimilar on both sides, average values were taken and analysis was done per patient and not per knee. This step was most relevant in patients who had KL Grade $1 \mathrm{OA}$ in one knee and Grade 2 in the other. We assumed that in patients with different stages $\mathrm{OA}$ in both knees, evaluation of effect of intervention would be difficult to differentiate. To overcome this bias and be as close to real life situation as possible, we decide to average the scores of both knees and analyze the results individualized to every single patient. The primary objective of the study was to compare improvement in pain, whereas the secondary outcomes were to compare the improvement of functional scores and incidence of complications.

\section{Statistical analysis}

Statistical analysis was performed using the SPSS version 22.0 software (IBM Corp., Armonk, NY, USA). Descriptive data were expressed in mean \pm standard deviation (SD) or median (min-max) for continuous variables and in number and percentage for categorical variables. Normality was assessed using a D'Agostino-Pearson test. Comparisons of the categorical variables between the study groups were performed using the Chi-square test and Fisher's exact test. Variation in the scores over different time periods in each study group were compared using repeated measures analysis of variance (ANOVA) with Bonferroni post-hoc test. Comparison of the scores between the three study groups at each time interval was performed using one-way ANOVA and Tukey post-hoc test. Comparison of the mean age, hemoglobin level, and platelet count between the three study groups were performed using one-way ANOVA. A $p$ value of $<0.05$ was considered statistically significant.

\section{RESULTS}

All patients completed the study and there were no protocol violations. All three groups were comparable to baseline demographic, clinical and disease characteristics (Table 1). The KL grades were the same on both sides in 51 patients, whereas they were dissimilar in 39 patients. All scores - mean VAS, IKDC Score, KOOS and TL score - were comparable between the three groups prior to intervention (Table 2). Within each group, all scores showed

\begin{tabular}{|c|c|c|c|c|c|c|c|}
\hline \multirow[b]{2}{*}{ Variables } & \multicolumn{2}{|c|}{ 1-dose group } & \multicolumn{2}{|c|}{ 2-dose group } & \multicolumn{2}{|c|}{ 3-dose group } & \multirow[b]{2}{*}{$p$} \\
\hline & $\%$ & Mean \pm SD & $\%$ & Mean \pm SD & $\%$ & Mean \pm SD & \\
\hline Age (year) & & $48.4 \pm 7.8$ & & $46.7 \pm 6.7$ & & $47.6 \pm 8.0$ & $0.75 \dagger$ \\
\hline $\begin{array}{l}\text { Sex } \\
\quad \text { Female }\end{array}$ & 76.7 & & 66.7 & & 83.3 & & $0.32 \ddagger$ \\
\hline Blood platelet count* & & $196 \pm 4$ & & $193 \pm 3$ & & $193 \pm 3$ & $0.93 \dagger$ \\
\hline $\begin{array}{l}\text { Kellgren-Lawrence Grade } \\
\text { Grade } 1\end{array}$ & 43.3 & & 48.3 & & 53.3 & & $0.68 \neq$ \\
\hline
\end{tabular}




\begin{tabular}{|c|c|c|c|c|}
\hline & 1-dose group & 2-dose group & 3-dose group & \\
\hline & Mean \pm SD & Mean \pm SD & Mean \pm SD & $p \dagger$ \\
\hline \multicolumn{5}{|l|}{ Visual Analog Scale } \\
\hline Pre-intervention & $7.7 \pm 1.0$ & $7.4 \pm 1.0$ & $7.6 \pm 1.0$ & 0.58 \\
\hline 6 weeks & $5.1 \pm 1.1$ & $5.0 \pm 1.0$ & $5.0 \pm 1.0$ & 0.85 \\
\hline 3 months & $3.0 \pm 1.2$ & $2.8 \pm 1.2$ & $2.7 \pm 1.0$ & 0.45 \\
\hline 6 months & $1.1 \pm 0.8$ & $1.0 \pm 0.8$ & $1.0 \pm 0.8$ & 0.65 \\
\hline 1 year & $3.7 \pm 1.0$ & $3.4 \pm 1.3$ & $1.5 \pm 1.3$ & $<0.001^{*}$ \\
\hline$p$ value $\neq$ & $<0.001^{*}$ & $<0.001^{*}$ & $<0.001^{*}$ & \\
\hline \multicolumn{5}{|l|}{ IKDC Score } \\
\hline Pre-intervention & $48.8 \pm 8.6$ & $50.3 \pm 7.4$ & $51.4 \pm 7.1$ & 0.42 \\
\hline 6 weeks & $57.4 \pm 8.0$ & $58.5 \pm 6.9$ & $59.9 \pm 6.5$ & 0.4 \\
\hline 3 months & $62.7 \pm 7.9$ & $63.7 \pm 6.4$ & $65.2 \pm 6.3$ & 0.37 \\
\hline 6 months & $68.9 \pm 8.2$ & $69.8 \pm 6.9$ & $71.5 \pm 6.4$ & 0.38 \\
\hline 1 year & $60.6 \pm 8.3$ & $61.8 \pm 6.9$ & $71.7 \pm 6.4$ & $<0.001^{*}$ \\
\hline$p$ value & $<0.001^{*}$ & $<0.001^{*}$ & $<0.001^{*}$ & \\
\hline \multicolumn{5}{|l|}{ KOOS } \\
\hline Pre-intervention & $49.0 \pm 7.5$ & $49.9 \pm 6.8$ & $50.8 \pm 7.0$ & 0.61 \\
\hline 6 weeks & $55.6 \pm 7.7$ & $56.4 \pm 6.7$ & $57.0 \pm 6.9$ & 0.73 \\
\hline 3 months & $62.1 \pm 7.0$ & $62.9 \pm 6.2$ & $63.6 \pm 6.4$ & 0.69 \\
\hline 6 months & $68.2 \pm 7.4$ & $69.4 \pm 6.4$ & $71.1 \pm 7.1$ & 0.58 \\
\hline 1 year & $59.1 \pm 7.3$ & $59.9 \pm 6.3$ & $69.3 \pm 6.7$ & $<0.001^{*}$ \\
\hline$p$ value & $<0.001^{*}$ & $<0.001^{*}$ & $<0.001^{*}$ & \\
\hline \multicolumn{5}{|c|}{ Tegner Lysholm knee score } \\
\hline Pre-intervention & $59.8 \pm 8.3$ & $60.7 \pm 6.7$ & $61.4 \pm 7.1$ & 0.69 \\
\hline 6 weeks & $67.0 \pm 8.3$ & $67.2 \pm 7.3$ & $67.9 \pm 7.1$ & 0.89 \\
\hline 3 months & $73.7 \pm 8.1$ & $74.7 \pm 7.3$ & $75.7 \pm 7.2$ & 0.61 \\
\hline 6 months & $79.5 \pm 8.9$ & $80.8 \pm 7.9$ & $81.5 \pm 8.2$ & 0.63 \\
\hline 1 year & $71.9 \pm 8.4$ & $72.3 \pm 7.9$ & $80.5 \pm 7.9$ & $<0.001^{*}$ \\
\hline$p$ value & $<0.001^{*}$ & $<0.001^{*}$ & $<0.001^{*}$ & \\
\hline
\end{tabular}

a statistically significant difference compared to baseline at all time points. The scores showed an improvement over six weeks, three and six months and tended to deteriorate at one year in single dose and double dose groups. In the triple dose group, the scores at one year were closer to that of six months, showing a tendency to sustain the scores achieved at six months (Table 2).

We also compared performance between the three groups at each time point of follow-up. There was no statistically significant difference between the mean scores between groups at six weeks, three months, and six months. However, at one year, the triple dose group had better outcome scores than the double and single dose groups which was statistically significant (Table 2).

\section{DISCUSSION}

The IA administration of PRP seems to play a definite role in treatment of early stages of $\mathrm{OA}$ knee. However, there seems to be many uncertainties regarding its clinical use such as optimal preparation, optimal dose, and need for repeat injections. ${ }^{3,5}$ A good number of studies with single dose of IA PRP injection showed a good initial response and deterioration of effect after six months of injection.,11,16 One of the strategies which came up to combat this problem was to increase the dose of PRP, and there is some evidence to suggest that multiple injections can improve the effectiveness of the procedure. ${ }^{6}$

In the literature, there are only six prospective, randomized trials comparing the dosage schedules in PRP in OA knee. ${ }^{7-12}$ Three of them had other 


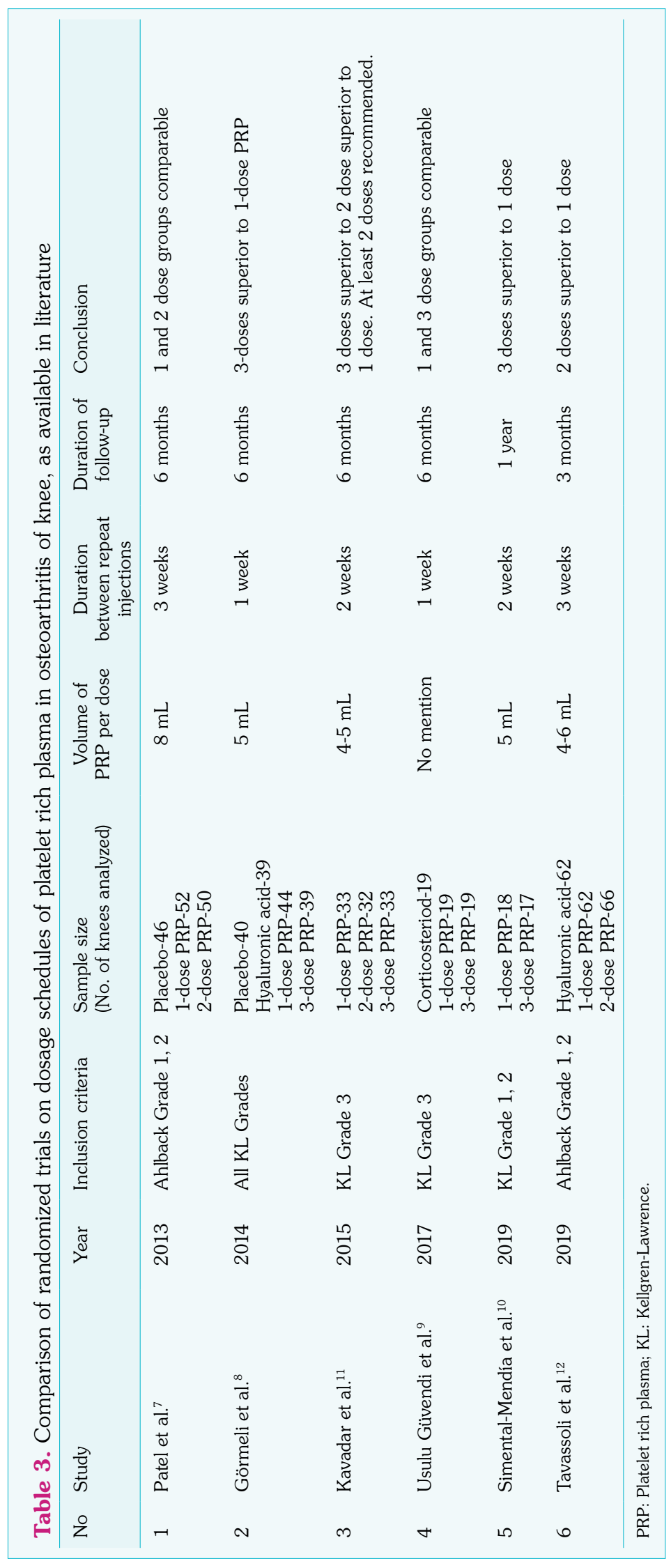


treatment arms such as hyaluronic acid ${ }^{8,12}$ and corticosteroid, ${ }^{9}$ and two of the studies had placebo controls. ${ }^{7,8}$ The studies differ widely in their methodology, duration of follow-up, and follow-up assessment and have come out with conflicting conclusions (Table 3). Similar to all previous authors, we did not find any serious complications in any group, except for transient increased in pain in 16 patients that settled within three days with oral paracetamol.-12

Our study design was based on previous works on the subject. We decided not to include placebo or steroid/hyaluronic acid arms as PRP was consistently superior to all of them in all available studies. The PRP seems to work better in early stages of stages of OA knee and, hence, we confined to selecting only $\mathrm{KL}$ 1-2 grades. Since OA is usually bilateral and the condition of both knees affect the functional scoring, we selected only bilateral cases to remove the bias assessment. All patients had injections on both sides simultaneously and both knees of the patients were randomized to the same arm to keep the assessment consistent. In the past, comparisons were made between one, two, and three doses of PRP, and we decided to include all three arms in a single study. The volume of PRP injected in one setting varied from 4 to $8 \mathrm{~mL},{ }^{7-11}$ we decided to stay at $4 \mathrm{~mL}$, consistent with previous studies. The gap between two injections varied between one to three weeks in published reports, ${ }^{7-11}$ we decided to stay in the middle and, thus, adopted two weeks.

The studies by Patel et al. ${ }^{7}$ ( 1 vs. 2 doses) and Uslu Güvendi et al. ${ }^{9}$ ( 1 vs. 3 doses) did not find any significant difference between single and multiple doses. The only available randomized study comparing three dosage schedules concluded that three doses were better than two doses which was again better than single dose. ${ }^{11}$ However, since the difference in the outcome between two and three doses was not significant, the study concluded recommending a minimum of two doses. The common factor between these three studies is that the follow-up period was only six months at which even our patients showed comparable results. When we extended the follow-up to one year, the three-dose group sustained the outcome measures better, although they also tended to deteriorate. In contrast, the study by Görmeli et al. ${ }^{8}$ showed superiority of three doses even at six months follow-up. More interestingly, the study by Tavassoli et al., ${ }^{12}$ showed superiority of two doses over one dose even at three months follow-up. ${ }^{12}$ Compared to available studies, our novelty is that we included single, double, and triple dose arms in early stages of OA knee with an extended follow-up of one year (Table 3).

Our study suggests that "multidose" means at least three doses, but not just two. However, we are unsure as to what is the explanation for this. If the outcome is just related to the volume of injection, increasing the volume of initial doses may reduce the number of doses required. Research in this direction has been initiated by a study in the guinea pig model by Chouhan et al. ${ }^{25}$ They compared single versus three doses and examined their histological effect and demonstrated that single dose of PRP had only short term anti-inflammatory effect on the synovium, whereas three doses resulted in sustained anti-inflammatory effect on the synovium with additional chondroprotective effect.

Our study is consistent with the findings of the retrospective study by Huang et al., ${ }^{13}$ where three doses showed a definite superiority to one or two doses at one year follow-up. All our scores (except for IKDC) showed a mild deterioration compared to those at six months, indicating that they may go down further. Unfortunately, Huang et al., ${ }^{13}$ did not analyze their scores at any time point before one year to confirm this pattern.

There are two important studies using three doses of PRP that followed the subjects longitudinally at least for two years. Filardo et al. ${ }^{24}$ administered three doses of PRP at three-week intervals and found a significant improvement in pain at two and six months of follow-up. However, the scores worsened progressively at 12 and 24 months, despite being significantly better than pre-treatment scores. At the end of the study, the authors concluded that scores were best maintained until nine months, although the benefit was significant even at the end of two years. In another study, Gobbi et al. ${ }^{16}$ demonstrated sustained improvement of scores at one year after three doses of monthly injection of PRP. Half of their patients received a second cycle of three doses of PRP injection. This 
group showed significant superiority to others at 18 months, but both groups showed deterioration of scores, which was comparable at two years. The authors concluded that the results of three doses of PRP at one year could be sustained by another cycle of injection. Although the scores were ill-sustained at two years, the procedure was worthwhile, since the improvement from baseline scores was significant.

Nonetheless, there are some limitations to the present study. Patients, rather than individual knees, were randomized to treatment arms. Although the results might have become more valid if the two sides of the same patient had been randomized to two different treatment arms, the study would have been cumbersome, raising ethical issues on two different treatment protocols on the same patient. In addition, although blinding the patient and surgeon would have added to the validity of conclusions, it would involve placebo injections which we were not ethical. Ultrasound-guided injections have been shown to be more accurate than blind injections in the literature $;^{26}$ however, we were unable to employ image guidance in our study. The follow-up of one year was also relatively short, particularly in the light of tendency of the scores to decline at the end of one year even in the three-dose group. These results can be considered as only preliminary. We believe that follow-up of at least two years is needed to make definite conclusions. Of note, our conclusions are only based on patient-reported scores. Along with PRP injections, we prescribed a regimen of life style modification, regular home-based exercises, and use of paracetamol as and when required. All of these must have had their share in the outcome scores and we are unsure how much the role of PRP exactly is. Validation of these results with radiographic and basic science correlation is the direction for future research.

In conclusion, IA administration of three doses of $4 \mathrm{~mL}$ of leucocyte poor-PRP yields superior outcome to one and two doses at the end of one year. Based on the literature findings and our study results where there is a tendency for the scores to deteriorate, repeat doses are probably needed to sustain the benefit achieved at one year. The timing and dosage of these repeat doses must be investigated in future studies.

\section{Acknowledgements}

We acknowledge the help rendered by Dr Sandhya Ramachandran (Head of the Dept of Pathology) and Dr Sai Kiran (Head of Blood Bank), Sri Sathya Sai Institute of Higher Medical Sciences - Prashanthigram, Puttaparthi, Andhra Pradesh, India and Dr Abhishek MB, Consultant Orthopaedic Surgeon, Sahyadri Narayan Multispeciality Hospital, Shivamogga, Karnataka India, in preparation and standardization of platelet rich plasma used in this study.

\section{Declaration of conflicting interests}

The authors declared no conflicts of interest with respect to the authorship and/or publication of this article.

\section{Funding}

The authors received no financial support for the research and/or authorship of this article.

\section{REFERENCES}

1. Stewart WF, Ricci JA, Chee E, Morganstein D, Lipton R. Lost productive time and cost due to common pain conditions in the US workforce. JAMA 2003;290:2443-54.

2. De La Mata J. Platelet rich plasma. A new treatment tool for the rheumatologist? Reumatol Clin 2013;9:166-71.

3. Delanois RE, Etcheson JI, Sodhi N, Henn RF 3rd, Gwam CU, George NE, et al. Biologic Therapies for the Treatment of Knee Osteoarthritis. J Arthroplasty 2019;34:801-13.

4. Sánchez M, Jorquera C, Sánchez P, Beitia M, García-Cano B, Guadilla J, et al. Platelet-rich plasma injections delay the need for knee arthroplasty: a retrospective study and survival analysis. Int Orthop 2020. [Online ahead of print]

5. Nguyen C, Lefèvre-Colau MM, Poiraudeau S, Rannou F. Evidence and recommendations for use of intraarticular injections for knee osteoarthritis. Ann Phys Rehabil Med 2016;59:184-9.

6. Vilchez-Cavazos F, Millán-Alanís JM, BlázquezSaldaña J, Álvarez-Villalobos N, Peña-Martínez VM, Acosta-Olivo CA, et al. Comparison of the Clinical Effectiveness of Single Versus Multiple Injections of Platelet-Rich Plasma in the Treatment of Knee Osteoarthritis: A Systematic Review and Meta-analysis. Orthop J Sports Med 2019;7:2325967119887116.

7. Patel S, Dhillon MS, Aggarwal S, Marwaha N, Jain A. Treatment with platelet-rich plasma is more effective than placebo for knee osteoarthritis: a prospective, double-blind, randomized trial. Am J Sports Med 2013;41:356-64.

8. Görmeli G, Görmeli CA, Ataoglu B, Çolak C, Aslantürk O, Ertem K. Multiple PRP injections are more effective than single injections and hyaluronic acid in knees with early osteoarthritis: a randomized, 
double-blind, placebo-controlled trial. Knee Surg Sports Traumatol Arthrosc 2017;25:958-65.

9. Uslu Güvendi E, Aşkin A, Güvendi G, Koçyiğit H. Comparison of Efficiency Between Corticosteroid and Platelet Rich Plasma Injection Therapies in Patients With Knee Osteoarthritis. Arch Rheumatol 2017;33:273-81.

10. Simental-Mendía M, Acosta-Olivo CA, HernándezRodríguez AN, Santos-Santos OR, de la Garza-Castro $\mathrm{S}$, Peña-Martínez VM, et al. Intraarticular injection of platelet-rich plasma in knee osteoarthritis: single versus triple application approach. Pilot study. Acta Reumatol Port 2019;44:138-44.

11. Kavadar G, Demircioglu DT, Celik MY, Emre TY. Effectiveness of platelet-rich plasma in the treatment of moderate knee osteoarthritis: a randomized prospective study. J Phys Ther Sci 2015;27:3863-7.

12. Tavassoli M, Janmohammadi N, Hosseini A, Khafri S, Esmaeilnejad-Ganji SM. Single- and double-dose of platelet-rich plasma versus hyaluronic acid for treatment of knee osteoarthritis: A randomized controlled trial. World J Orthop 2019;10:310-26.

13. Huang PH, Wang CJ, Chou WY, Wang JW, Ko JY. Short-term clinical results of intra-articular PRP injections for early osteoarthritis of the knee. Int $\mathrm{J}$ Surg 2017;42:117-22.

14. Altman R, Asch E, Bloch D, Bole G, Borenstein $\mathrm{D}$, Brandt $\mathrm{K}$, et al. Development of criteria for the classification and reporting of osteoarthritis. Classification of osteoarthritis of the knee. Diagnostic and Therapeutic Criteria Committee of the American Rheumatism Association. Arthritis Rheum 1986;29:1039-49.

15. Luyten FP, Bierma-Zeinstra S, Dell'Accio F, Kraus VB, Nakata K, Sekiya I, et al. Toward classification criteria for early osteoarthritis of the knee. Semin Arthritis Rheum 2018;47:457-63.

16. Gobbi A, Lad D, Karnatzikos G. The effects of repeated intra-articular PRP injections on clinical outcomes of early osteoarthritis of the knee. Knee Surg Sports Traumatol Arthrosc 2015;23:2170-7.
17. Dohan Ehrenfest DM, Rasmusson L, Albrektsson T. Classification of platelet concentrates: from pure platelet-rich plasma (P-PRP) to leucocyte- and plateletrich fibrin (L-PRF). Trends Biotechnol 2009;27:158-67.

18. DeLong JM, Russell RP, Mazzocca AD. Platelet-rich plasma: the PAW classification system. Arthroscopy 2012;28:998-1009.

19. Thomas KS, Muir KR, Doherty M, Jones AC, O'Reilly SC, Bassey EJ. Home based exercise programme for knee pain and knee osteoarthritis: randomised controlled trial. BMJ 2002;325:752.

20. Reed MD, Van Nostran W. Assessing pain intensity with the visual analog scale: a plea for uniformity. J Clin Pharmacol 2014;54:241-4.

21. Irrgang JJ, Anderson AF, Boland AL, Harner CD, Kurosaka M, Neyret $\mathrm{P}$, et al. Development and validation of the international knee documentation committee subjective knee form. Am J Sports Med 2001;29:600-13.

22. Roos EM, Lohmander LS. The Knee injury and Osteoarthritis Outcome Score (KOOS): from joint injury to osteoarthritis. Health Qual Life Outcomes 2003;1:64.

23. Lysholm J, Tegner Y. Knee injury rating scales. Acta Orthop 2007;78:445-53.

24. Filardo G, Kon E, Buda R, Timoncini A, Di Martino A, Cenacchi $A$, et al. Platelet-rich plasma intra-articular knee injections for the treatment of degenerative cartilage lesions and osteoarthritis. Knee Surg Sports Traumatol Arthrosc 2011;19:528-35.

25. Chouhan DK, Dhillon MS, Patel S, Bansal T, Bhatia A, Kanwat H. Multiple Platelet-Rich Plasma Injections Versus Single Platelet-Rich Plasma Injection in Early Osteoarthritis of the Knee: An Experimental Study in a Guinea Pig Model of Early Knee Osteoarthritis. Am J Sports Med 2019;47:2300-7.

26. Bum Park Y, Ah Choi W, Kim YK, Chul Lee S, Hae Lee J. Accuracy of blind versus ultrasound-guided suprapatellar bursal injection. J Clin Ultrasound 2012;40:20-5. 